\title{
Comprehensive analysis of the IncRNA-miRNA-mRNA ceRNA network in aortic dissection
}

Fengwen Xie, Jichun Liu

Department of Cardiovascular Surgery, The First Affiliated Hospital of Nanchang University, Nanchang, China

Submitted: 16 April 2020

Accepted: 19 June 2020

Arch Med Sci

DOI: https://doi.org/10.5114/aoms.2020.97354

Copyright $\odot 2020$ Termedia \& Banach

\section{Abstract}

Introduction: Aortic dissection (AD) is a critical cardiovascular system disease caused by blood in the aortic cavity penetrating into the middle layer of the aortic wall through the intimal rift and extending along the long axis of the aorta. Studies have shown that long noncoding RNAs (IncRNAs) can bind to microRNAs (miRNAs) as competing endogenous RNAs (ceRNAs) and further affect the levels of target mRNAs. The purpose of this study was to construct and analyse the IncRNA-miRNA-mRNA ceRNA network in AD.

Material and methods: We first downloaded the AD expression data of five normal samples and seven AD samples from the Gene Expression Omnibus (GEO) database (GSE52093) and identified differentially expressed mRNAs (DEmRNAs) and IncRNAs (DElncRNAs). Gene Ontology (GO) and Kyoto Encyclopedia of Genes and Genomes (KEGG) pathway analyses of DEmRNAs were performed using the DAVID and KOBAS online databases. The ceRNA network was constructed by using the miRcode, miRDB, miRTarBase, and TargetScan databases and visualised by Cytoscape v3.6.1. In addition, a protein-protein interaction (PPI) network of DEmRNAs was also constructed.

Results: In total, 13 IncRNAs and 472 mRNAs were identified as significantly differentially expressed between the $A D$ and normal samples $(|\log F C|>1.0$, false discovery rate $(F D R)<0.05)$. In addition, seven IncRNAs, 18 miRNAs, and 48 mRNAs contributed to the construction of the ceRNA network.

Conclusions: We confirmed the DElncRNAs and DEmRNAs in AD and constructed a IncRNA-miRNA-mRNA ceRNA network by using bioinformatics methods, which may provide a novel perspective for the diagnosis of, and potential therapeutic targets for, AD.

Key words: aortic dissection, long noncoding RNA, competitive endogenous RNA, Gene Expression Omnibus.

\section{Introduction}

Aortic dissection (AD) is a critical cardiovascular system disease caused by blood in the aortic cavity penetrating into the middle layer of the aortic wall through the intimal rift and extending along the long axis of the aorta. The cause of $A D$ is mainly related to hypertension [1]. In addition, genetics is also a very important factor. Current research shows that congenital genetic diseases such as Marfan syndrome, Eller-Danlow syndrome, Loeys-Dietz syndrome, and bicuspid aortic valve (BAV) can affect the growth of connective tissue, resulting in aortic dissection, especially Stanford type A aortic dissection [2, 3]. Epidemiological studies show that approximately 3-4 cases per 100,000 people develop acute

\author{
Corresponding author: \\ Jichun Liu \\ Department of \\ Cardiovascular Surgery \\ The First Affiliated \\ Hospital of \\ Nanchang University \\ Nanchang, China \\ E-mail: \\ liujichun9999@126.com
}


aortic dissection every year [4]. Currently, the main treatment methods include drug therapy, intracavitary isolation therapy, classic surgery, and hybrid surgery, but morbidity and mortality are still high [5]. A possible molecular mechanism of $A D$ is an imbalance in extracellular matrix (ECM) protein turnover and depletion of vascular smooth muscle cells (VSMCs) [6-8]. However, research on the molecular mechanism of AD is still limited.

Long noncoding RNA (InCRNA) refers to a class of RNA molecules longer than 200 nt that are widely present in the nucleus and cytoplasm of eukaryotic cells [9]. LncRNAs do not encode proteins but can be involved in many physiological and pathological processes through various mechanisms [10-13]. MicroRNA (miRNA) is a type of noncoding endogenous small-molecule single-stranded RNA with a length of 22 nt [14]. MiRNAs suppress the expression of target genes by partially complementing the miRNA response elements (MREs) in mRNA, and they participate in a series of important biological processes, including cell development, proliferation, apoptosis, death, differentiation, and fat metabolism [15]. In $A D$, miRNAs can further affect the homeostasis of arterial smooth muscle and ECM by affecting the synthesis of signalling pathway molecules and related substances in cells, thereby affecting the function and structure of the aorta [16-18]. The competitive endogenous RNA (ceRNA) hypothesis proposes that IncRNAs cannot only directly regulate the expression of target genes but may also affect the number of miRNA molecules by binding to the core seed sequences of miRNAs and further affecting the levels of target mRNAs [19]. ceRNAs are widely involved in important biological activity regulatory processes, such as inflammation, apoptosis, and differentiation [20--22].

Numerous studies have shown that this regulatory mechanism is also active in many cardiovascular diseases, such as myocardial hypertrophy, myocardial infarction, atherosclerosis, and atrial fibrillation [23-26]. Currently, however, research on the role and mechanism of ceRNA in $A D$ is still lacking. The purpose of this study is to use bioinformatics methods to construct and analyse the IncRNA-miRNA-mRNA ceRNA network in $A D$ by reanalysing the gene expression profile of GSE52093 [27]. These results may provide useful information regarding the diagnosis of, and potential therapeutic targets for, AD.

\section{Material and methods}

\section{Microarray data}

Gene expression profiles of GSE52093 were obtained from the Gene Expression Omnibus (GEO) database (http://www.ncbi.nlm.nih.gov/ geo/). GSE52093 is publicly available and can be downloaded free of charge with confirmation that all relevant informed consent has been obtained. This dataset includes five normal ascending aorta samples and seven Stanford type A aortic dissection aorta samples. All the samples were analysed using an Illumina HumanHT-12 v4.0 Expression BeadChip (USA).

\section{Identification of differentially expressed mRNAs (DEmRNAs) and IncRNAs (DElncRNAs)}

Raw data were obtained from the GEO database and preprocessed by log2 transformation. Then, the data were normalised by the limma package [28] in $R$ statistical software. Linear models and the empirical Bayes method (limma) were used to analyse and identify DElncRNAs and DEmRNAs between the normal samples and $A D$ samples from the GEO database. The Benjamini and Hochberg method was applied to correct the $p$-value to the false discovery rate (FDR), and the cut-off criteria were $|\log F C|>1$ and FDR $<0.05$. In addition, mRNA and IncRNA annotation was performed using the Encyclopedia of DNA Elements (ENCODE) with ENSEMBL.

\section{GO and KEGG pathway analysis}

We used the DAVID (https://david.ncifcrf.gov/) online database for $\mathrm{GO}$ enrichment analysis of DEmRNAs, which included biological processes (BPs), cellular components (CCs), and molecular functions (MFs). The KOBAS online database (http://kobas.cbi.pku.edu.cn/) was used for KEGG pathway enrichment analysis. Records with an adjusted $p$-value $<0.05$ and enrichment $>2.0$ were considered to have statistical significance. The $R$ package "ggplot2" (version 3.3.0) was used for visualisation.

\section{Construction of the ceRNA and PPI networks}

The flowchart of ceRNA network construction is shown in Figure 1. First, the miRcode database (www.mircode.org/) was used to predict the IncRNA-miRNA interactome. Second, miRTarBase, miRDB, and TargetScan were used to predict the miRNA-mRNA interactome. The target mRNAs and DEmRNAs were then intersected. Finally, the ceRNA network was constructed by combining the IncRNA-miRNA interactions and miRNA-mRNA interactions, and visualised by Cytoscape v3.6.1. In addition, a protein-protein interaction (PPI) network of DEmRNAs in the ceRNA network was constructed using the Search Tool for the Retrieval of Interacting Genes (STRING). The parameter of interactive relationships was set up with high confidence $>0.700$. 


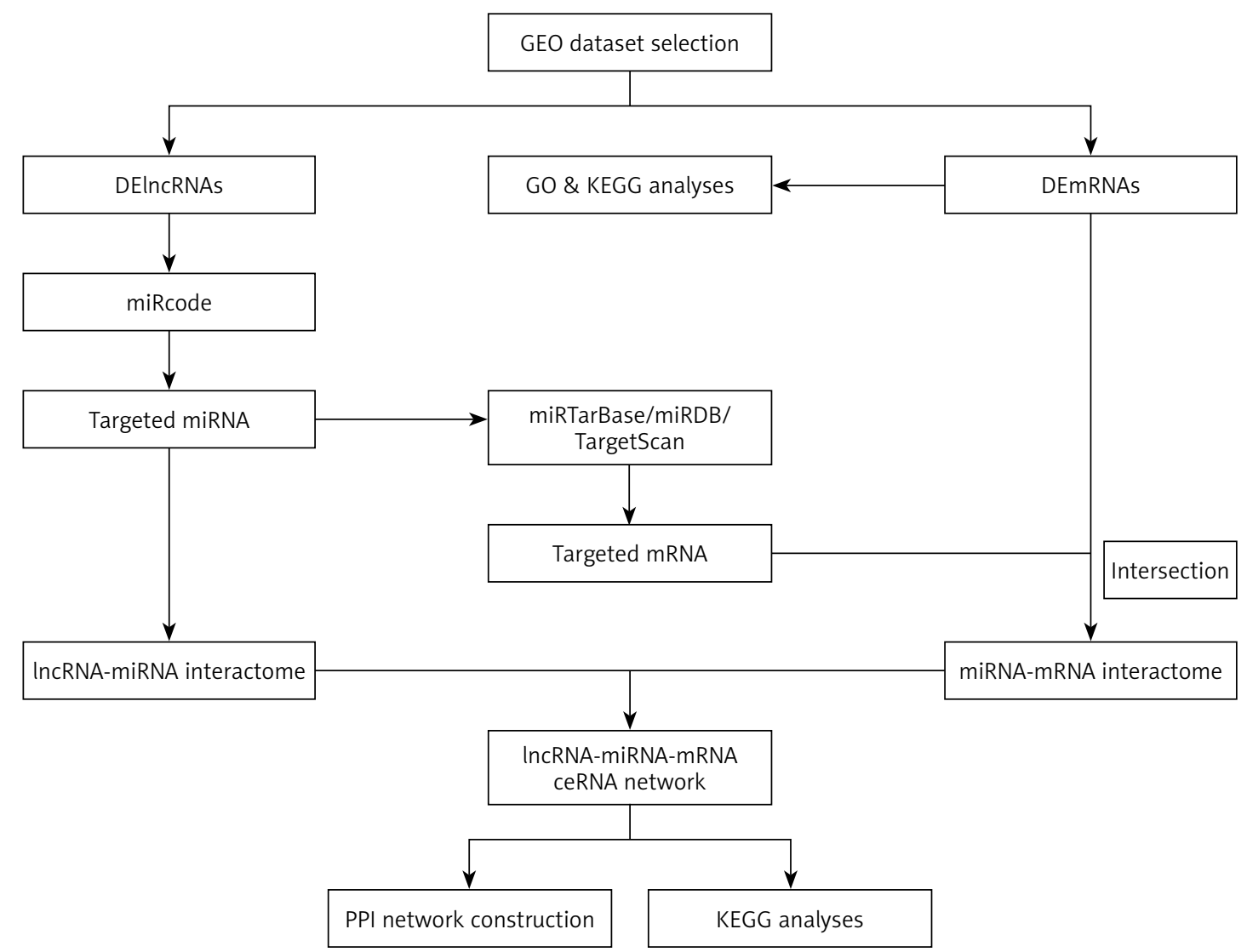

Figure 1. Flow chart of the construction of the ceRNA network in AD

\section{Statistical analysis}

Numerical data were expressed as the mean \pm standard deviation (SD) of at least three measurements. Statistical comparisons between groups of normalised data were performed using the $t$-test or Mann-Whitney U-test according to the test condition. A $p$-value $<0.05$ was considered statistically significant with a $95 \%$ confidence level.

\section{Results}

\section{Identification of DEmRNAs and DElncRNAs in $A D$}

Based on the cut-off criteria of $|\log F C|<1$ and FDR $<0.05$, we found 485 differentially expressed genes between the AD and normal aortic samples, as shown in the volcano map in Figure $2 \mathrm{~A}$. We identified 13 DElncRNAs, five of which were upregulated, while eight were downregulated (Table I). In addition, 472 mRNAs were identified as differentially expressed, 296 of which were upregulated, while 176 were downregulated. The heatmaps of DElncRNAs and DEmRNAs are shown in Figures $2 \mathrm{~B}$ and $\mathrm{C}$, respectively.

\section{Enrichment analysis of DEmRNAs in AD}

To further explore the biological functions of the 472 DEmRNAs in $A D$, we performed $G O$ and
KEGG enrichment analyses. The results show that the top enriched GO terms for BPs were chromosome segregation, DNA replication, nuclear chromosome segregation, mitotic nuclear division, and DNA-dependent DNA replication. The most enriched CCs were spindle, chromosomal region, chromosome, centromeric region, myofibril, and contractile fibre. Finally, the most enriched MFs were actin binding, structural constituent of muscle, and histone kinase activity (Figure $3 \mathrm{~A}$ ). The KEGG enrichment analysis revealed that the DEmRNAs were significantly enriched in the following pathways: metabolic pathways, focal adhesion, cell cycle, PI3K-Akt signalling pathway, DNA replication, and regulation of actin cytoskeleton (Figure $3 \mathrm{~B}$ ).

\section{Construction of the ceRNA and PPI networks in $A D$}

To further understand how IncRNAs regulate mRNA expression by binding to miRNAs in AD, a ceRNA network was constructed. Eighteen miRNAs were predicted to interact with seven DElnCRNAs by using the miRcode database (Table II). A total of 1124 miRNA-targeted mRNAs based on the 18 miRNAs were predicted through the miRDB, miRTarBase, and TargetScan databases. We selected 48 mRNAs among the 472 DEmRNAs 
A

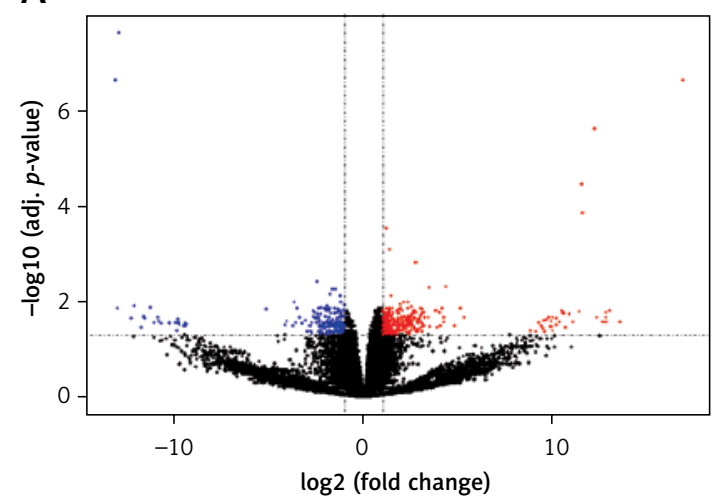

C
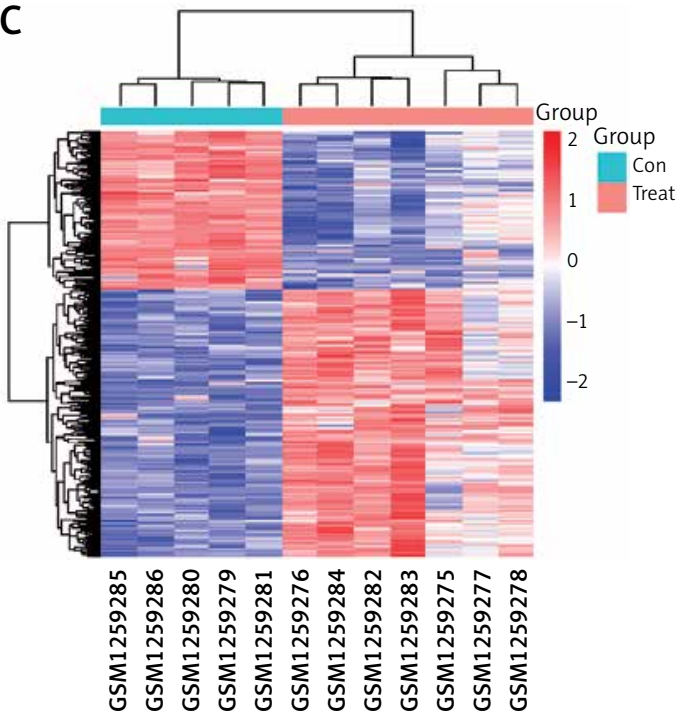

B
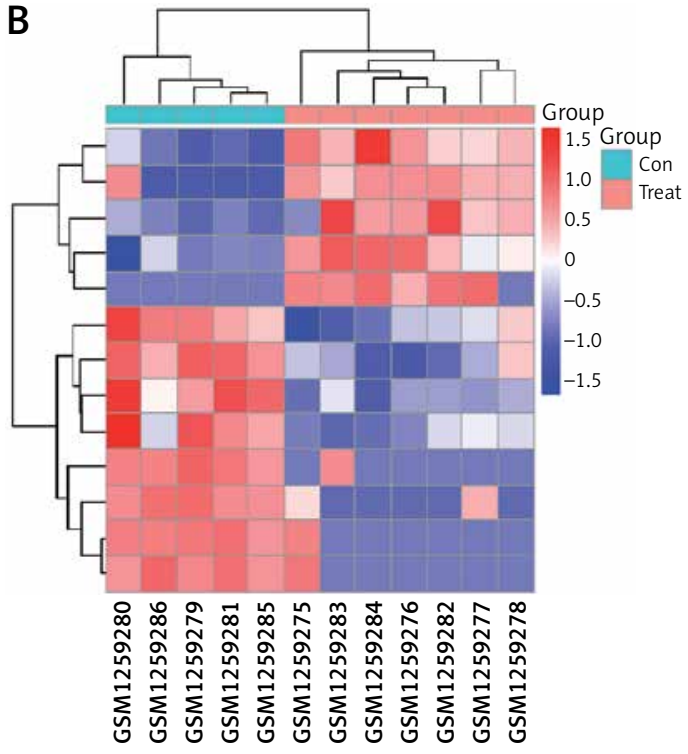

Figure 2. Differentially expressed genes between AD patients and normal controls. A - The volcano plot of the differentially expressed genes. The $\mathrm{X}$-axis is - $\log 10$ (adjusted $p$-value), while the $Y$-axis is log2FC. Black dots indicate non-differently expressed genes. The red dots indicate upregulated genes, while the blue dots indicate downregulated genes. B - Heatmap of differentially expressed IncRNAs. C - Heatmap of differentially expressed mRNAs. B, C - The $\mathrm{X}$-axis is the sample serial number, and the $\mathrm{Y}$-axis is the differentially expressed genes. Red blocks represent upregulation, and green blocks represent downregulation

Table I. Dysregulated IncRNAs between normal controls and aortic dissection patients

\begin{tabular}{|llll|}
\hline InCRNA & Expression change & LogFC(AD/N) & Adj. $p$-value \\
\hline AATBC & Upregulation & 9.25238493 & 0.028727158 \\
\hline C15orf32* $^{*}$ & Upregulation & 9.10185404 & 0.042884568 \\
\hline FBXL19-AS1* & Upregulation & 1.588913174 & 0.017449507 \\
\hline CHEK2P2* $^{*}$ & Upregulation & 1.394313623 & 0.028336018 \\
\hline LINC00467 $^{*}$ & Upregulation & 1.110905614 & 0.044152098 \\
\hline NUP210P1* & Downregulation & -1.394044025 & 0.041875981 \\
\hline LINC01252 $^{*}$ & Downregulation & -1.814109151 & 0.041887661 \\
\hline CARMN & Downregulation & -1.845765285 & 0.02165766 \\
\hline UCA1* & Downregulation & -2.424119434 & 0.018942266 \\
\hline RGPD4-AS1 & Downregulation & -9.503621401 & 0.032708119 \\
\hline SMG1P5 & Downregulation & -9.79031617 & 0.027508735 \\
\hline INGX* & Downregulation & -9.854377146 & 0.023345615 \\
\hline LINC01114 & Downregulation & -10.78663878 & 0.025996043 \\
\hline
\end{tabular}

$A D$ - aortic dissection, $N$ - normal sample. *Represents the IncRNAs taking part in the ceRNA network. 
A

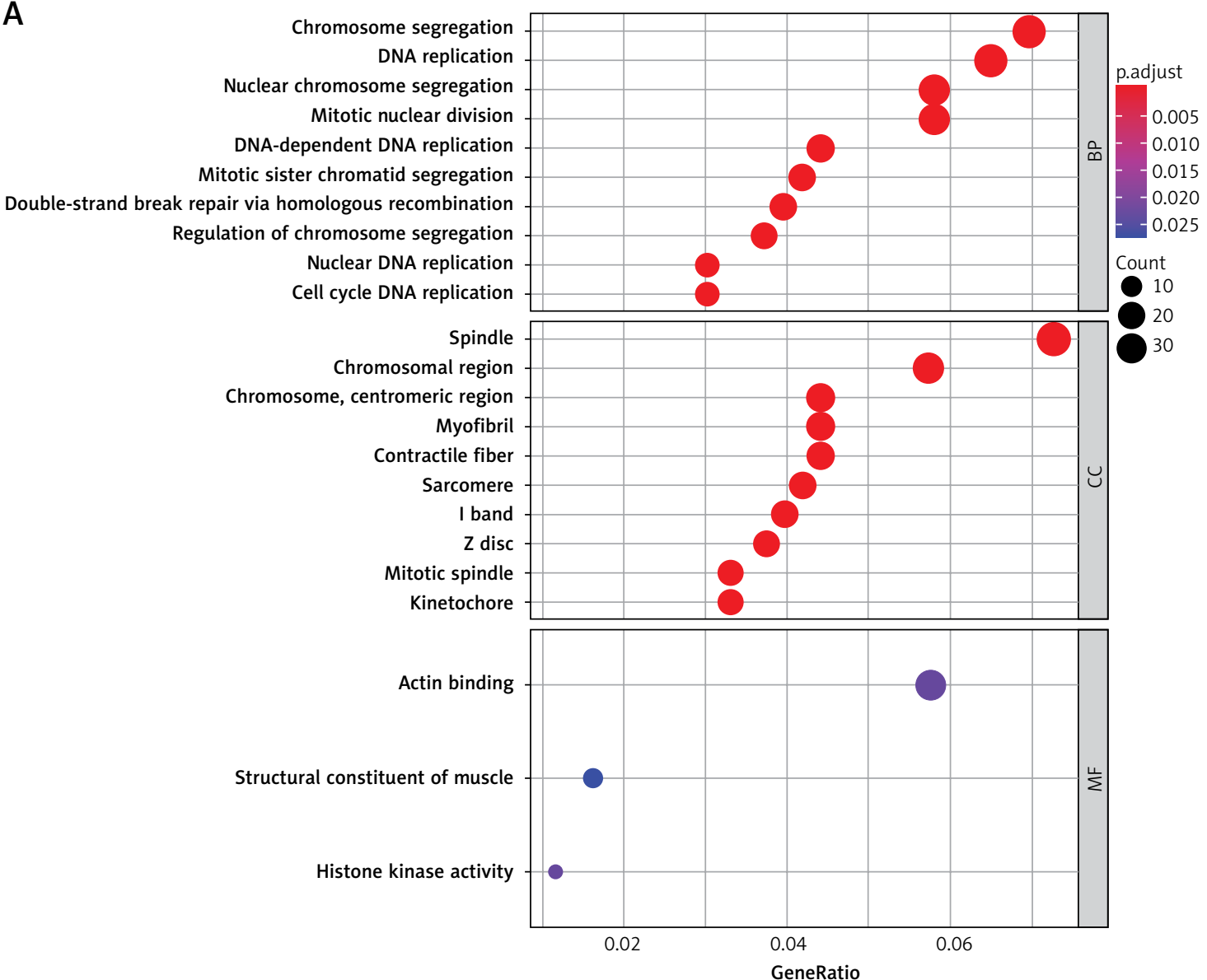

Figure 3. Enrichment analysis of differentially expressed mRNAs. A - Bubble plot of the GO pathway enrichment analysis of differentially expressed mRNAs

GO - Gene Ontology, KEGG - Kyoto Encyclopedia of Genes and Genomes.

that were identified as miRNA targets of mRNAs (Figure $4 \mathrm{~A}$, Table III). Finally, the ceRNA network was constructed based on the seven IncRNAs, 18 miRNAs, and 48 mRNAs and visualised by using Cytoscape v3.6.1 (Figure 5). In addition, most KEGG pathways enriched for DEmRNAs in the ceRNA network were associated with regulation of the actin cytoskeleton, the prolactin signalling pathway, the TGF- $\beta$ signalling pathway, Fc $\gamma$ R-mediated phagocytosis, and the PI3K-Akt signalling pathway (Figure 4 B). The PPI network constructed for the 48 mRNAs provided 15 genes with a high confidence of 0.700 (Figure 4 C).

\section{Discussion}

$A D$ is caused by a variety of factors inflicting damage on the aortic intima, and then blood flows into the aortic intima and tears the media, thereby forming an intravascular pseudocavity, which can cause aorta rupture and seriously threaten the lives of patients. Treatment options are limited [1]. In-depth research on the pathogenesis of $A D$ can help us to further develop screening tools, identify different risk categories, and discover po- tential therapeutic targets, which is of great clinical significance. A previous study detected the expression profiles of seven $A D$ samples and five normal samples in GSE52093 but focused mainly on a subset of DEmRNAs [29, 30]. In this study, we focused not only on DEmRNAs but also DElncRNAs. At the same time, we constructed a ceRNA network and conducted a comprehensive bioinformatics analysis of DEmRNAs and DElncRNAs in the ceRNA network by reanalysing the published microarray data of GSE52093.

In our study, we found 472 DEmRNAs in $A D$ samples compared to normal samples. The results of the GO pathway analysis for BPs showed that DEmRNAs are related to chromosome segregation and DNA replication, which in turn are associated with the characteristics of cells. Previous studies have shown that chromosome segregation is related to the development of aortic dissection [31], which is consistent with the results of our GO analysis. These results show that the physiological process of arterial wall cells play a key role in maintaining the stability of the aorta and warrants further exploration. In addition to vascular smooth 
B

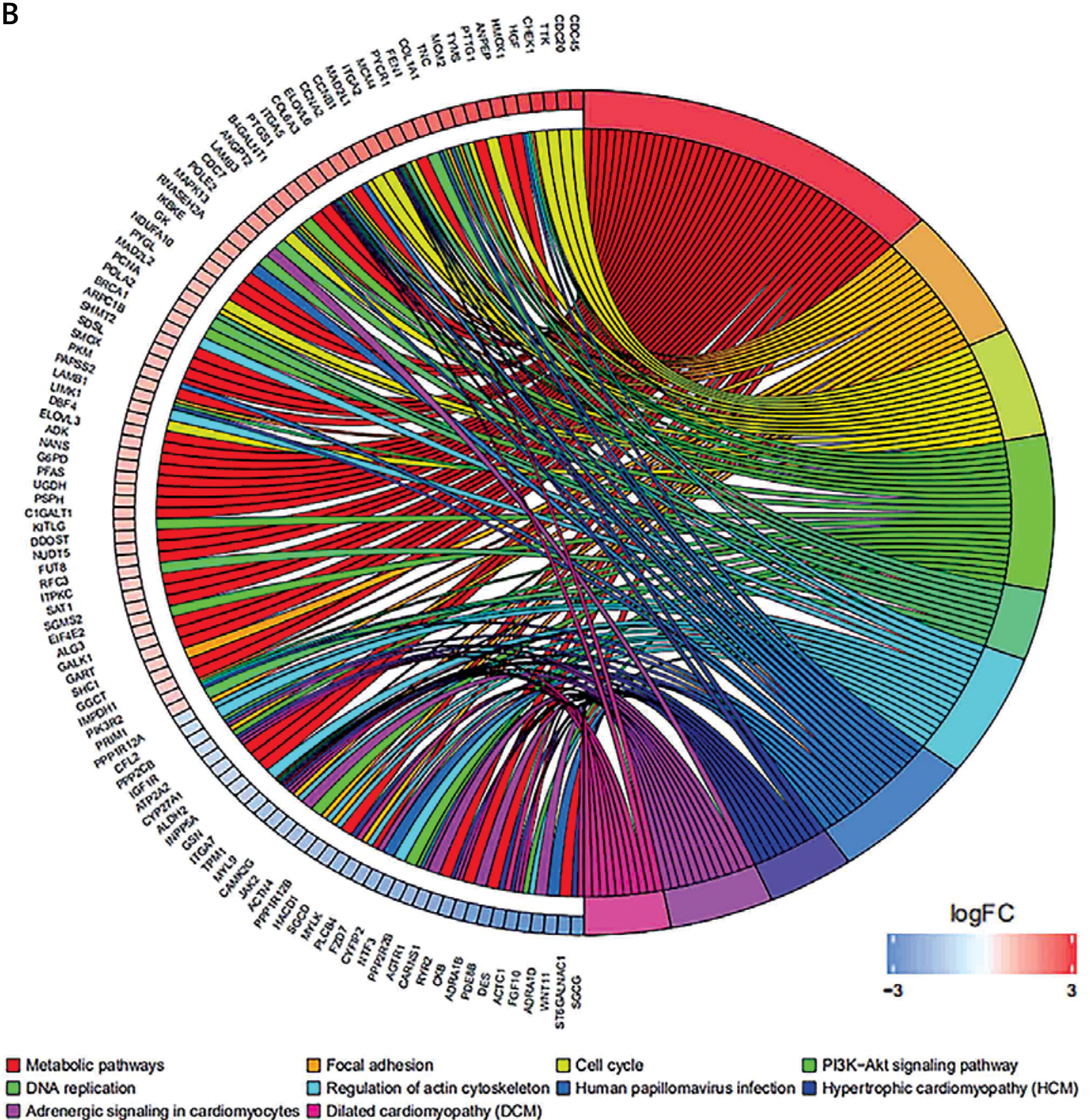

Figure 3. Cont. B - Circle plot of the KEGG enrichment analysis of differentially expressed mRNAs GO - Gene Ontology, KEGG - Kyoto Encyclopedia of Genes and Genomes.

Table II. IncRNAs and specific targeted miRNAs in the ceRNA network

\begin{tabular}{|lc|}
\hline IncRNA & miRNA \\
\hline LINC00467 & $\begin{array}{r}\text { hsa-miR-27a-3p, hsa-miR-107 } \\
\hline \text { C15orf32 }\end{array}$ \\
\hline $\begin{array}{r}\text { NUP210P1 } \\
\text { hsa-miR-1244, hsa-miR-17-5p, hsa-miR-20b-5p, hsa-miR-107, hsa-miR-338-3p, } \\
\text { hsa-miR-33a-3p }\end{array}$ \\
\hline UCA1 & hsa-miR-135a-5p, hsa-miR-206, hsa-miR-1297, hsa-miR-107, hsa-miR-129-5p \\
\hline INGX & hsa-miR-17-5p, hsa-miR-20b-5p, hsa-miR-22-3p, hsa-miR-24-3p, hsa-miR-129-5p \\
\hline CHEK2P2 & hsa-miR-146b-5p, hsa-miR-338-3p \\
\hline FBXL19-AS1 & $\begin{array}{c}\text { hsa-miR-135a-5p, hsa-miR-142-3p, hsa-miR-17-5p, hsa-miR-20b-5p, hsa-miR-216b-5p, } \\
\text { hsa-miR-22-3p, hsa-miR-24-3p, hsa-miR-1297, hsa-miR-107, hsa-miR-129-5p }\end{array}$ \\
\hline
\end{tabular}



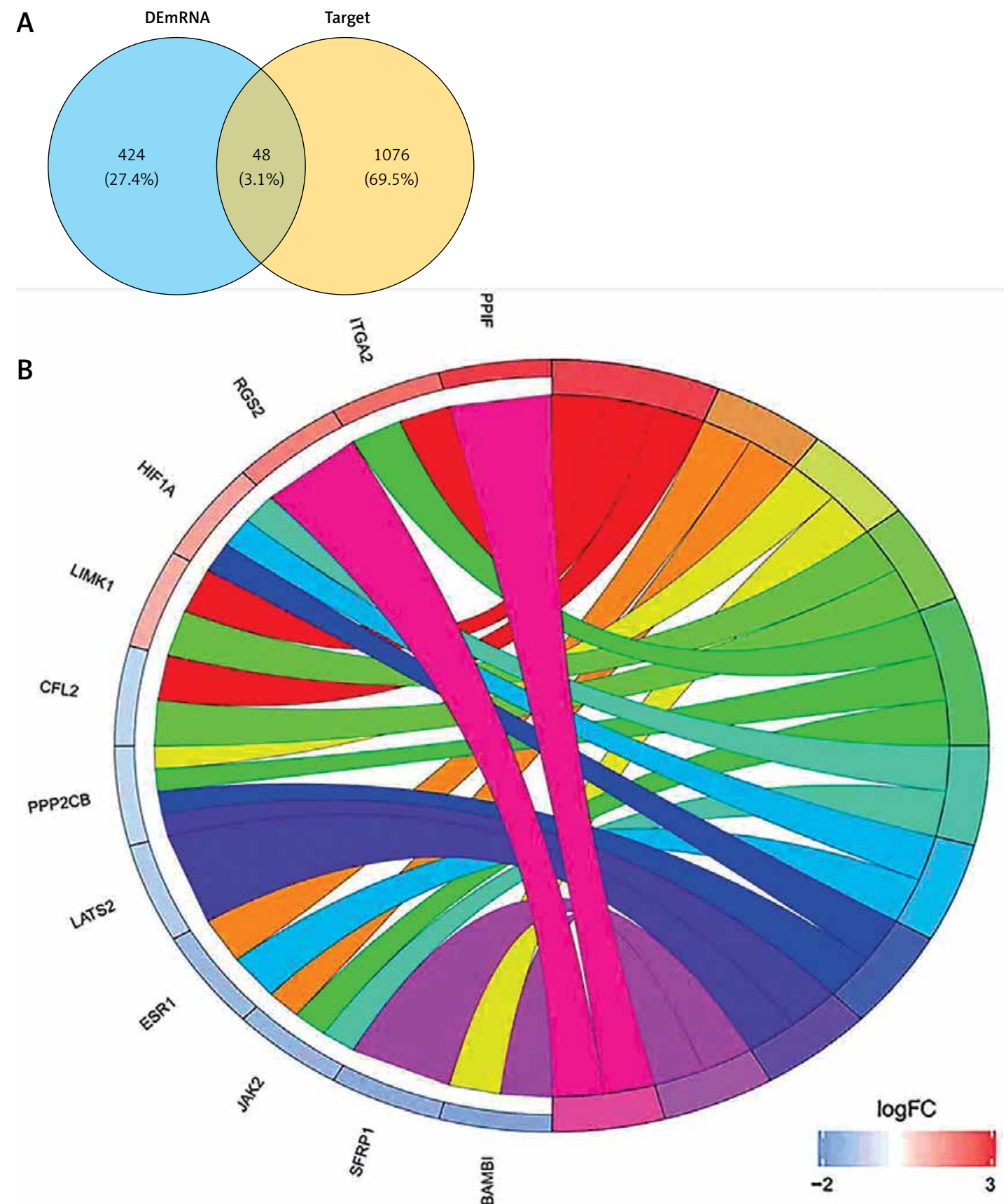

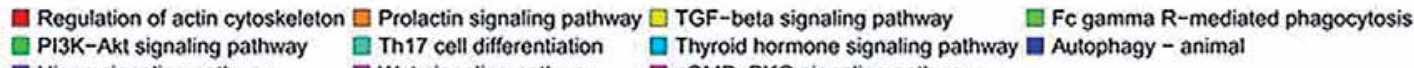
Hippo signaling pathway
믈 Wignaling pathway

cGMP-PKG signaling pathway

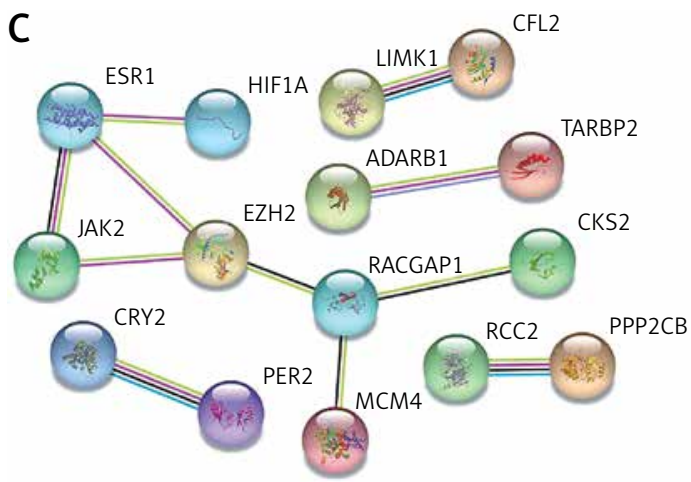

Figure 4. mRNAs in the ceRNA network. A - Venn diagram of differentially expressed mRNAs in the ceRNA network. B - KEGG pathways enriched by mRNAs in the ceRNA network. $\mathbf{C}$ - The protein-protein interaction network constructed for mRNAs involved in the ceRNA network 
Table III. miRNAs and specific targeted mRNAs in the ceRNA network

\begin{tabular}{|c|c|}
\hline miRNA & mRNA \\
\hline hsa-miR-107 & ITGA2, RIMS3, TARBP2, LATS2 \\
\hline hsa-miR-1244 & SAT1 \\
\hline hsa-miR-129-5p & SESN3, SOX4, SORBS2, REEP1 \\
\hline hsa-miR-1297 & CKS2 \\
\hline hsa-miR-135a-5p & JAK2, VLDLR \\
\hline hsa-miR-142-3p & $\begin{array}{c}\text { CPEB2, INPP5A, C5orf24, } \\
\text { HMGA1 }\end{array}$ \\
\hline hsa-miR-146b-5p & IRAK 1 \\
\hline hsa-miR-17-5p & $\begin{array}{l}\text { CFL2, HIF1A, CRY2, ADARB1, } \\
\text { SEMA4B, PDLIM5, POLQ, } \\
\text { SESN3, LIMK1, PKD2, SOX4 }\end{array}$ \\
\hline hsa-miR-206 & G6PD, SFRP1 \\
\hline hsa-miR-20b-5p & $\begin{array}{l}\text { POLQ, SOX4, CFL2, LIMK1, } \\
\text { SEMA4B, SESN3, HIF1A, } \\
\text { BAMBI, CRIM1, CRY2 }\end{array}$ \\
\hline hsa-miR-216b-5p & PPP2CB, ZDHHC9, MCM4 \\
\hline hsa-miR-217 & $\mathrm{EZH} 2, \mathrm{GPC} 5$ \\
\hline hsa-miR-22-3p & $\begin{array}{r}\text { C5orf24, ESR1, RGS2, } \\
\text { RCC2, KCTD10 }\end{array}$ \\
\hline hsa-miR-24-3p & MLEC, PER2, MT1E, PLIN3 \\
\hline hsa-miR-27a-3p & PPIF, HNRNPF \\
\hline hsa-miR-301b-3p & SOX4, CFL2, ESR1, RACGAP1 \\
\hline hsa-miR-338-3p & PFAS \\
\hline hsa-miR-33a-3p & SESN3, CPEB2, LMNB2 \\
\hline
\end{tabular}

muscle contraction and ECM-receptor interactions, which have been reported as two potential pathways in $A D[6-8,30]$, the KEGG results show that $A D$ is also associated with metabolic pathways and regulation of the actin cytoskeleton. Studies have found that metabolite products related to the choline metabolic pathway are differentially expressed in $A D$ and can be used as biomarkers for the diagnosis of $A D$ and evaluations of therapeutic effects [32]. Studies show that regulation of the actin cytoskeleton can affect VSMC migration by regulating the migration process [33]. Therefore, we believe that metabolic pathways and regulation of the actin cytoskeleton may play important roles in $A D$ and are worth studying. In addition, we found that MCM4 as a DEmRNA is not only a hub gene in $A D$, according to a previous study [29], but also contributed to the construction of the ceRNA network in this study, indicating that MCM4 may play an important role in AD. MCM4 is a member of the MCM family, which is essential for initiating replication of eukaryotic genomes and may be involved in the repair of DNA double-strand breaks, the formation of replication forks, and the assembly of other replication-related proteins [34]. Research shows that MCM4 is involved in the proliferation of multiple tumours, suggesting that MCM4 is an important indicator of cell proliferation and warrants further study $[35,36]$.

A total of 18 miRNAs were predicted to interact with the seven IncRNAs in the ceRNA network of $A D$ in our study. Among them, hsa-miR-17-5p, hsamiR-20b-5p, and hsa-miR-22-3p interrelated with five or more protein-coding genes, which may play more important roles in the ceRNA network. These aberrantly expressed miRNAs are also involved in the pathological processes of other diseases. For example, studies have shown that hsa-miR-17-5p promotes apoptosis induced by oxidative stress by targeting Stat3, hsa-miR-20b-5p has been observed to promote lung cancer cell proliferation and migration by targeting BTG3, and hsa-miR22-3p was reported to inhibit the proliferation and migration of arterial smooth muscle cells by targeting HMGB1 in arteriosclerosis obliterans [3739]. However, the specific mechanisms of these miRNAs in AD have rarely been studied.

In the ceRNA network, IncRNAs can regulate mRNA expression through competitive binding with miRNAs, thereby participating in the regulation of cell biology. We found 18 DElncRNAs in AD, seven of which participated in the ceRNA network. C15orf32 was the most upregulated IncRNA in the ceRNA network, indicating that $\mathrm{C} 15$ orf32 plays an important role in AD. Few studies on C15orf32 are available to date. According to limited studies, C15orf32 is a neurodevelopment-related gene and is thought to be related to Alzheimer's disease [40]. In addition, another notable IncRNA in the ceRNA network was FBXL19-AS1, which interacted with as many as 10 miRNAs. FBXL19-AS1 is an oncogene that can promote cancer cell proliferation and metastasis in breast cancer and lung cancer [41, 42]. Studies have found that IncRNAs are not only associated with human cancers but can also affect the proliferation of VSMCs [43-46]. Therefore, we speculate that FBXL19-AS1 may also have this function, and jointly with regulation of the actin cytoskeleton affect the biological processes of VSMCs and thus participate in the formation of $A D$. However, research on the function of IncRNA as a miRNA sponge in AD is still lacking.

Some limitations of this study must be acknowledged. First, the sample size in this study was not large. Second, the samples were almost all from males (only one AD sample was from a female), which may introduce a gender bias. Third, the genotype and phenotype data of patients was lacking in the study. Last, the bioinformatics analysis in our study is just the first step. Therefore, it is necessary for us to validate the findings by collecting more clinical samples and conducting more in-depth experiments in the future.

In conclusion, we identified 13 DElncRNAs and 472 DEmRNAs in AD and constructed a lncRNA-miRNA-mRNA ceRNA network for the first 


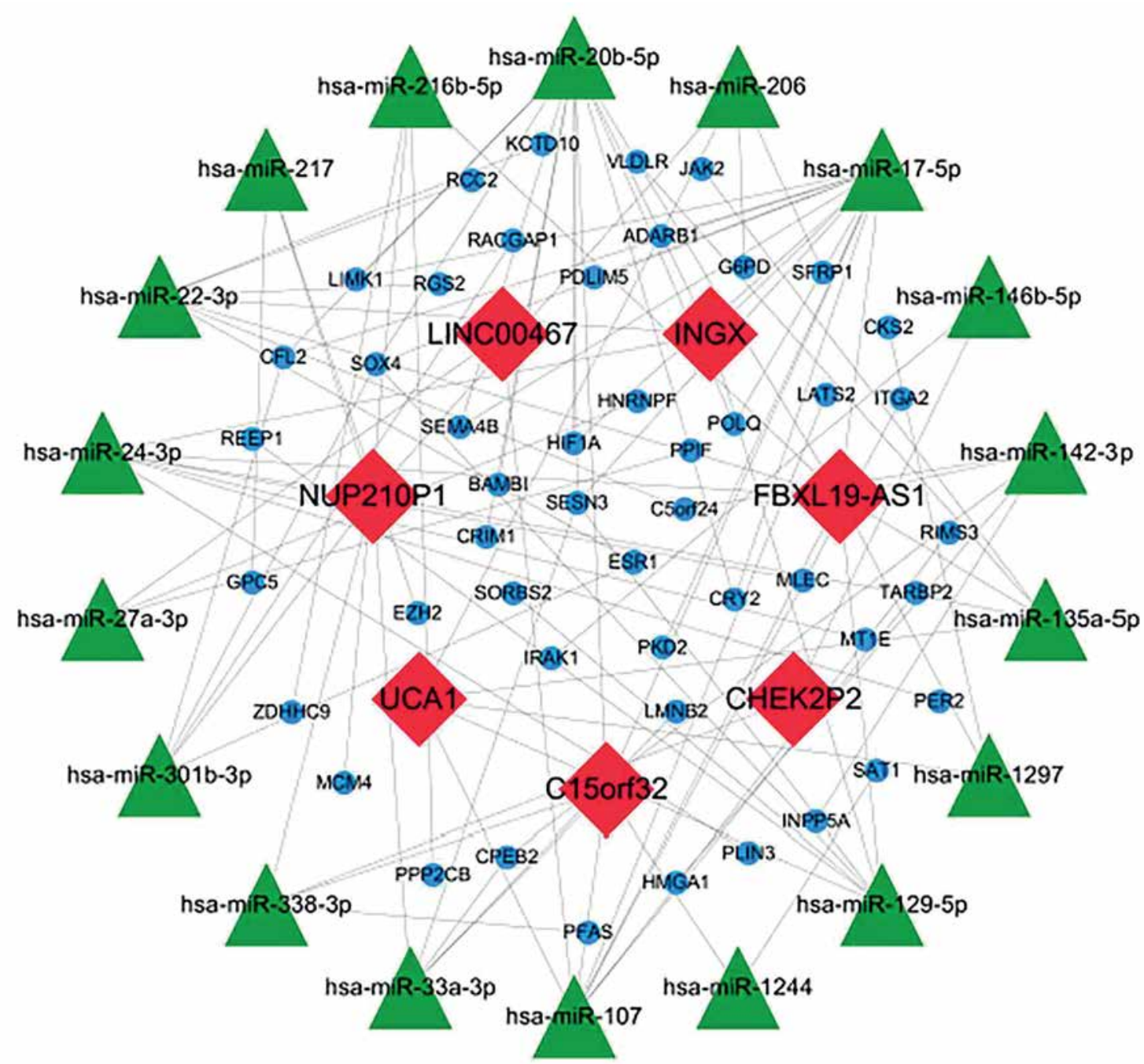

Figure 5. ceRNA network in AD. Diamonds represent IncRNAs, triangles represent miRNAs, and circles represent protein-coding genes. Grey edges indicate IncRNA-miRNA-mRNA interactions

time. These findings may provide new insights into the pathogenesis of $A D$ and a novel perspective for further developing screening tools, identifying different risk categories, and discovering potential therapeutic targets for AD.

\section{Acknowledgments}

We sincerely thank the Gene Expression Omnibus (GEO) for the RNA expression data on aortic dissection.

\section{Conflict of interest}

The authors declare no conflict of interest.

\section{References}

1. Riambau V, Bockler D, Brunkwall J, et al. Editor's choice - management of descending thoracic aorta diseases: clinical practice guidelines of the European Society for Vascular Surgery (ESVS). Eur J Vasc Endovasc Surg 2017; 53: 4-52.
2. Gawinecka J, Schonrath F, von Eckardstein A. Acute aortic dissection: pathogenesis, risk factors and diagnosis. Swiss Med Wkly 2017; 147: w14489.

3. Homme JL, Aubry MC, Edwards WD, et al. Surgical pathology of the ascending aorta: a clinicopathologic study of 513 cases. Am J Surg Pathol 2006; 30: 1159-68.

4. Pacini D, Di Marco L, Fortuna D, et al. Acute aortic dissection: epidemiology and outcomes. Int I Cardiol 2013; 167: 2806-12.

5. Sidloff D, Choke E, Stather P, et al. Mortality from thoracic aortic diseases and associations with cardiovascular risk factors. Circulation 2014; 130: 2287-94.

6. Zhao Z, Wang Y, Li S, et al. HSP90 inhibitor 17-DMAG effectively alleviated the progress of thoracic aortic dissection by suppressing smooth muscle cell phenotypic switch. Am J Transl Res 2019; 11: 509-18.

7. Cheng J, Zhou X, Jiang X, Sun T. Deletion of ACTA2 in mice promotes angiotensin II induced pathogenesis of thoracic aortic aneurysms and dissections. J Thorac Dis 2018; 10: 4733-40.

8. An Z, Qiao F, Lu Q, et al. Interleukin-6 downregulated vascular smooth muscle cell contractile proteins via ATG4B-mediated autophagy in thoracic aortic dissection. Heart Vessels 2017; 32: 1523-35. 
9. Xu R, Han Y. Long non-coding RNA FOXF1 adjacent non-coding developmental regulatory RNA inhibits growth and chemotherapy resistance in non-small cell lung cancer. Arch Med Sci 2019; 15: 1539-46.

10. Yu P, Guo J, Li J, Chen W, Zhao T. Co-expression network analysis revealing the key IncRNAs in diabetic foot ulcers. Arch Med Sci 2019; 15: 1123-32.

11. Necsulea A, Soumillon M, Warnefors M, et al. The evolution of IncRNA repertoires and expression patterns in tetrapods. Nature 2014; 505: 635-40.

12. Jiang L, Hong L, Yang W, et al. Co-expression network analysis of the IncRNAs and mRNAs associated with cervical cancer progression. Arch Med Sci 2019; 15: 754-64

13. Liu X, Wang M, Cui Y. LncRNA TP73-AS1 interacted with miR-141-3p to promote the proliferation of non-small cell lung cancer. Arch Med Sci 2019; 15: 1547-54.

14. Fila M, Pawlowska E, Blasiak J. Mitochondria in migraine pathophysiology - does epigenetics play a role? Arch Med Sci 2019; 15: 944-56.

15. Kaneko $\mathrm{H}$, Terasaki $\mathrm{H}$. Biological involvement of microRNAs in proliferative vitreoretinopathy. Transl Vis Sc Technol 2017; 6: 5.

16. Shan H, Zhang Y, Lu Y, et al. Downregulation of miR-133 and miR-590 contributes to nicotine-induced atrial remodelling in canines. Cardiovasc Res 2009; 83: 465-72.

17. Li Y, Huang J, Jiang $Z$, et al. MicroRNA-145 regulates platelet-derived growth factor-induced human aortic vascular smooth muscle cell proliferation and migration by targeting CD40. Am J Transl Res 2016; 8: 1813-25.

18. Kimura N, Futamura K, Arakawa M, et al. Gene expres sion profiling of acute type A aortic dissection combined with in vitro assessment. Eur J Cardiothorac Surg 2017; 52: 810-7

19. Salmena L, Poliseno L, Tay Y, Kats L, Pandolfi PP. A ceRNA hypothesis: the Rosetta Stone of a hidden RNA language? Cell 2011; 146: 353-8.

20. Wang WT, Ye H, Wei PP, et al. LncRNAs H19 and HULC, activated by oxidative stress, promote cell migration and invasion in cholangiocarcinoma through a ceRNA manner. J Hematol Oncol 2016; 9: 117.

21. Li D, Yang M, Liao A, et al. Linc00483 as ceRNA regulates proliferation and apoptosis through activating MAPKs in gastric cancer. J Cell Mol Med 2018; 22: 3875-86.

22. Guo Z, Cao Y. An IncRNAmiRNAmRNA ceRNA network for adipocyte differentiation from human adipose-derived stem cells. Mol Med Rep 2019; 19: 4271-87.

23. Wang K, Liu F, Zhou LY, et al. The long noncoding RNA CHRF regulates cardiac hypertrophy by targeting miR489. Circ Res 2014; 114: 1377-88.

24. Liang $\mathrm{H}$, Pan Z, Zhao X, et al. LncRNA PFL contributes to cardiac fibrosis by acting as a competing endogenous RNA of let-7d. Theranostics 2018; 8: 1180-94.

25. Shan K, Jiang Q, Wang XQ, et al. Role of long non-coding RNA-RNCR3 in atherosclerosis-related vascular dysfunction. Cell Death Dis 2016; 7: e2248.

26. Li Z, Wang X, Wang W, et al. Altered long non-coding RNA expression profile in rabbit atria with atrial fibrillation: TCONS_00075467 modulates atrial electrical remodeling by sponging miR-328 to regulate CACNA1C. J Mol Cell Cardiol 2017; 108: 73-85.

27. Pan S, Wu D, Teschendorff AE, et al. JAK2-centered interactome hotspot identified by an integrative network algorithm in acute Stanford type A aortic dissection. PLoS One 2014; 9: e89406

28. Ritchie ME, Phipson B, Wu D, et al. limma powers differential expression analyses for RNA-sequencing and microarray studies. Nucleic Acids Res 2015; 43: e47.
29. Wang W, Wang T, Wang Y, et al. Integration of gene expression profile data to verify hub genes of patients with Stanford A aortic dissection. Biomed Res Int 2019; 2019: 3629751.

30. Jiang T, Si L. Identification of the molecular mechanisms associated with acute type A aortic dissection through bioinformatics methods. Braz J Med Biol Res 2019; 52: e8950.

31. Erhart P, Brandt T, Straub BK, et al. Familial aortic disease and a large duplication in chromosome 16p13.1. Mol Genet Genom Med 2018; 6: 441-5.

32. Ren Y, Tang Q, Liu W, et al. Serum biomarker identification by mass spectrometry in acute aortic dissection. Cell Physiol Biochem 2017; 44: 2147-57.

33. Tang DD, Gerlach BD. The roles and regulation of the actin cytoskeleton, intermediate filaments and microtubules in smooth muscle cell migration. Respir Res 2017; 18: 54.

34. Yardimci H, Walter JC. Prereplication-complex formation: a molecular double take? Nat Struct Mol Biol 2014 21: 20-5.

35. Kikuchi J, Kinoshita I, Shimizu Y, et al. Minichromosome maintenance (MCM) protein 4 as a marker for proliferation and its clinical and clinicopathological significance in non-small cell lung cancer. Lung Cancer 2011; 72 229-37.

36. Huang XP, Zhang X, Su XD, et al. Expression and significance of MCM4 in esophageal cancer. Ai Zheng 2007; 26: 96-9.

37. Du W, Pan Z, Chen X, et al. By targeting Stat3 microRNA-17-5p promotes cardiomyocyte apoptosis in response to ischemia followed by reperfusion. Cell Physiol Biochem 2014; 34: 955-65.

38. Peng L, Li S, Li Y, et al. Regulation of BTG3 by microRNA-20b-5p in non-small cell lung cancer. Oncol Lett 2019; 18: 137-44.

39. Huang SC, Wang M, Wu WB, et al. Mir-22-3p inhibits arterial smooth muscle cell proliferation and migration and neointimal hyperplasia by targeting HMGB1 in arteriosclerosis obliterans. Cell Physiol Biochem 2017; 42: 2492-506.

40. Porcelli S, Crisafulli C, Donato L, et al. Role of neurodevelopment involved genes in psychiatric comorbidities and modulation of inflammatory processes in Alzhei mer's disease. J Neurol Sci 2016; 370: 162-6.

41. Dong G, Pan T, Zhou D, et al. FBXL19-AS1 promotes cell proliferation and inhibits cell apoptosis via miR-876-5p/ FOXM1 axis in breast cancer. Acta Biochim Biophys Sin 2019; 51: 1106-13.

42. Jiang O, Cheng L, Ma D, Zhao Y. FBXL19-AS1 exerts on cogenic function by sponging miR-431-5p to regulate RAF1 expression in lung cancer. Biosci Rep 2019; 39: pii: BSR20181804.

43. Lei $H$, Gao $Y, X u X$. LncRNA TUG1 influences papillary thyroid cancer cell proliferation, migration and EMT formation through targeting miR-145. Acta Biochim Biophys $\operatorname{Sin} 2017$; 49: 588-97.

44. Li FP, Lin DQ, Gao LY. LncRNA TUG1 promotes proliferation of vascular smooth muscle cell and atherosclerosis through regulating miRNA-21/PTEN axis. Eur Rev Med Pharmacol Sci 2018; 22: 7439-47.

45. Wei GH, Wang X. IncRNA MEG3 inhibit proliferation and metastasis of gastric cancer via p53 signaling pathway. Eur Rev Med Pharmacol Sci 2017; 21: 3850-6.

46. Wang M, Li C, Zhang Y, et al. LncRNA MEG3-derived miR361-5p regulate vascular smooth muscle cells proliferation and apoptosis by targeting ABCA1. Am J Transl Res 2019; 11: 3600-9. 